\title{
Relationship between urine lipid peroxidation, anthropometric parameters and parameters associated with goitre formation in school-age children
}

\author{
Arkadiusz Zygmunt ${ }^{1,2}$, Jan Stepniak ${ }^{3}$, Katarzyna Wojciechowska-Durczynska $a^{1,2}$, \\ Kinga Krawczyk-Rusiecka ${ }^{1,2}$, Andrzej Lewinski, ${ }^{1,2}$, Malgorzata Karbownik-Lewinska²,3
}

\author{
${ }^{1}$ Department of Endocrinology and Metabolic Diseases, Medical University of Lodz, \\ Lodz, Poland \\ 2Polish Mother's Memorial Hospital - Research Institute, Lodz, Poland \\ ${ }^{3}$ Department of Oncological Endocrinology, Medical University of Lodz, Lodz, Poland
}

Submitted: 17 May 2017

Accepted: 18 July 2017

Arch Med Sci 2018; 14, 1: 30-37

DOI: https://doi.org/10.5114/aoms.2018.72240

Copyright @ 2017 Termedia @ Banach

\section{Abstract}

Introduction: Oxidative stress has been implicated in the normal ageing process and the pathogenesis of several diseases, including goitre. The aim of the study was to evaluate the relationship between urine lipid peroxidation (LPO) and anthropometric parameters as well as the parameters associated with goitre formation in children.

Material and methods: The subjects included 172 healthy children (93 girls and 79 boys) aged $8-15$, divided into 4 age groups - group I (8-9 years), group II (10-11 years), group III (12-13 years) and group IV (14-15 years) - and into 2 groups based on the BSA: the BSA-1 group $\left(\leq 0.55 \mathrm{~m}^{2}\right)$ and the BSA-2 group (>0.55 $\mathrm{m}^{2}$ ).

Results: The value of LPO was the highest in group I but the difference between the groups was not statistically significant $(p=0.074)$. In the BSA-1 group, the LPO was higher than in the BSA-2 group $(12.75 \pm 6.90 \mathrm{nmol} /$ $\mathrm{ml}$ and $10.79 \pm 4.86 \mathrm{nmol} / \mathrm{ml}$, respectively; $p=0.023)$. We found a weak, negative linear correlation between LPO and age $(r=-0.216 ; p<0.005)$, body mass $(r=-0.153 ; p<0.05)$, height $(r=-0.152 ; p<0.05)$ and BSA $(r=-0.151 ; p<0.05)$.

Conclusions: Anthropometric parameters of school-age children independently of age are negatively associated with oxidative damage to membrane lipids, whereas factors promoting goitrogenesis do not contribute to this process.

Key words: oxidative stress, iodine, thyroid gland.

\section{Introduction}

Animal cells produce reactive oxygen species (ROS), including free radicals, which participate in the metabolic processes [1, 2]. Overproduction of ROS, due to the action of either external or internal factors, may cause increased damage to macromolecules, including membrane lipids [3, 4]. Typically, ROS are neutralised by the antioxidant defence system, but in some cases, the oxidants may exceed the capacity of these mechanisms and disrupt the redox balance, leading to the condition known as oxidative stress [5].

\author{
Corresponding author: \\ Andrzej Lewinski \\ Department of \\ Endocrinology and \\ Metabolic Diseases \\ Medical University \\ of Lodz \\ 281/289 Rzgowska St \\ 93-338 Lodz, Poland \\ Phone: +48 422711141 \\ Fax: +48 422711140 \\ E-mail: alewin@csk.umed. \\ lodz.pl
}


Oxidative stress has been implicated in the normal ageing process and the pathogenesis of several diseases, including cancer $[3,4]$.

Reactive oxygen species are required for hormone synthesis in the thyroid gland $[6,7]$ but oxidative stress also creates conditions required for thyroid cell proliferation [8]; thus increased oxidative stress is associated with goitre formation $[9,10]$.

Measuring the oxidative stress status and its effects on biomolecules may help to assess the risk of developing thyroid diseases, including goitre formation.

It should be emphasized that studies on oxidoreduction reactions are being used to address a variety of issues in both experimental and clinical medicine [11, 12].

Biological membranes, being a source of polyunsaturated fatty acids (PUFAs), are susceptible to ROS. The action of free radicals on PUFAs results in lipid peroxidation (LPO), which causes structural changes in cellular membranes and impairs the cellular function. In our previous studies LPO has frequently been measured in the blood $[13,14]$. Determination of the urinary LPO level allows one to avoid invasive procedures for taking biological samples, which is especially important in children.

The present study is a continuation of the survey conducted among school-age children on iodine deficiency [15].

The aim of the study was to evaluate the potential relationship between urine LPO and anthropometric parameters as well as the parameters associated with goitre formation in children.

\section{Material and methods}

The study was approved by the Ethics Committee at the Polish Mother's Memorial Hospital - Research Institute, Lodz, Poland.

\section{Subjects}

The subjects included 172 healthy children (93 girls and 79 boys) aged 8-15, recruited from the Primary School in Opoczno, a town in the centre of Poland, about 80 kilometres south-east of Lodz.

The children were divided into 4 age groups: group I (8-9 years), group II (10-11 years), group III (12-13 years) and group IV (14-15 years). The characteristics of the examined population are presented in Table I.

The children were also divided into two groups according to body surface area (BSA): the BSA-1 group included children whose BSA was equal to or less than $0.55 \mathrm{~m}^{2}$; the BSA-2 group included children with BSA > $0.55 \mathrm{~m}^{2}$.

Parents of all children included in the study gave written consent to the participation of their children in the study. The Ethics Committee approved the protocol.

\section{Methods}

The height and the body mass of children were measured using standard anthropometric techniques [16]. For the measurements children took off their shoes and wore light indoor clothing.

The heights were recorded to the nearest millimetre, and the body mass was recorded to the nearest $100 \mathrm{~g}$. BSA was calculated from the following formula: $B S A=W^{0.425} \times H^{0.725} \times 71.84 \times 10^{-4}$, where: $W=$ weight $(\mathrm{kg}) ; H=$ height $(\mathrm{cm})$.

Body mass index (BMI) was calculated from the formula: $\mathrm{BMI}=W / H^{2}$, where: $W=$ weight $(\mathrm{kg})$; $H=$ height (m).

The calculated value of BMI was then compared with the values on the centile charts appropriate for the age and sex.

\section{Sample collection and analytical determinations}

Urine samples were collected from each child in the morning, and then they were frozen at $-70^{\circ} \mathrm{C}$ until the analytical determinations of the LPO products and urine iodine concentration (UIC).

\section{LPO assay}

The concentrations of malondialdehyde + 4-hydroxyalkenals (MDA+4-HDA), as the index of LPO, were measured in urine, using an LPO-586 kit, purchased from Enzo Life Science (Farmingdale, NY, USA). The urine $(200 \mu \mathrm{l})$ was mixed with $650 \mu$ l of a methanol:acetonitrile $(1: 3, \mathrm{v} / \mathrm{v})$ solution, containing a chromogenic reagent, $\mathrm{N}$-meth$\mathrm{yl}$-2-phenylindole, and vortexed. After adding $150 \mu \mathrm{l}$ of methanesulfonic acid (15.4 M), incubation was carried out at $45^{\circ} \mathrm{C}$ for $40 \mathrm{~min}$. The reaction between MDA+4-HDA and N-methyl-2-phenylindole yields a chromophore, which is spectrophotometrically measured at the absorbance of $586 \mathrm{~nm}$, using a solution of 4-hydroxynonenal $(10 \mathrm{mM})$ as the standard. The level of LPO was expressed as the amount of MDA+4-HDA (nmol) per $1 \mathrm{ml}$ of serum.

The manufacturer, i.e. Enzo Life Science (Farmingdale, NY, USA), confirmed that the LPO-586 kit might be used for LPO measurement in urine.

In order to determine iodide concentration, the modified catalytic method by Sandell and Kolthoff was used [17].

\section{Statistical analysis}

Prior to performing any other statistical analysis, the normality of the distribution of examined parameters was evaluated using the one-sample Kolmogorov-Smirnov test. The data were statistically analyzed, using the non-parametric test for independent groups (Mann-Whitney rank sum test), Kruskal-Wallis one way analysis of variance 


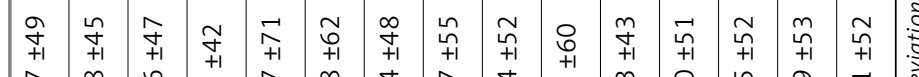

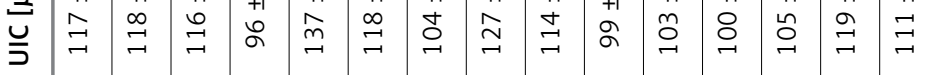

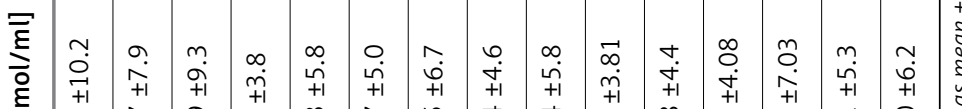

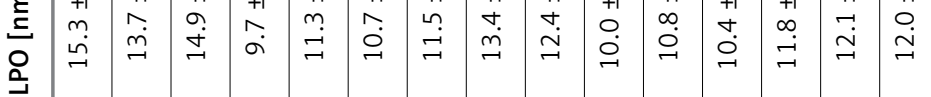

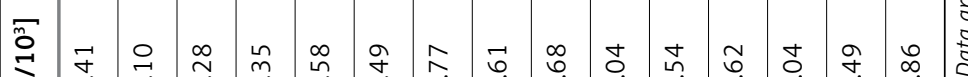

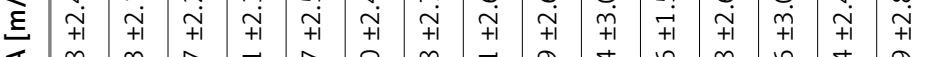

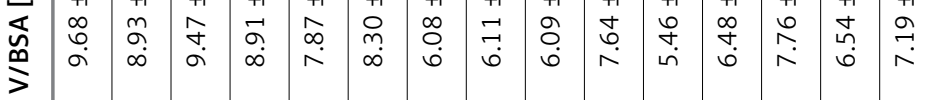

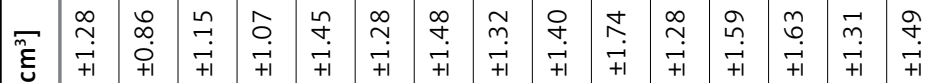

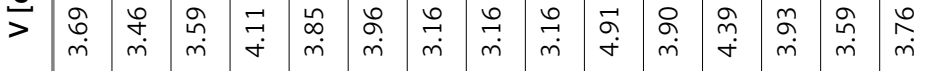

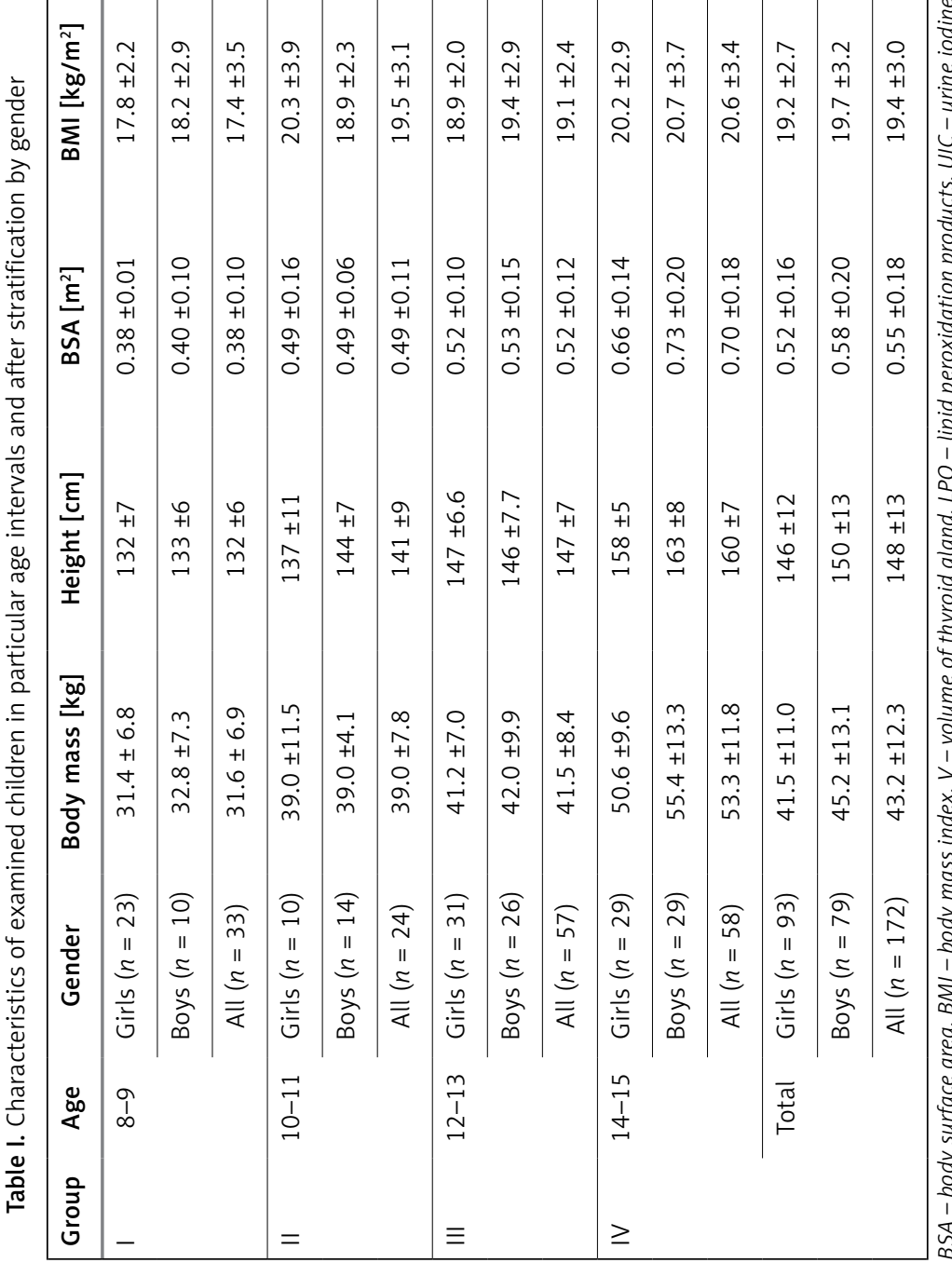


on ranks, followed by Dunn's test, $\chi^{2}$ analysis and Pearson correlation. In all analyses, statistical significance was accepted at a value of $p<0.05$.

Data processing, statistical analyses and figures were performed by using SigmaPlot 12.3 (Systat Software, Inc., San Jose, CA, USA) and Excel (Microsoft Corp., Redmond, WA, USA).

\section{Results}

\section{LPO products}

Although the value of LPO was the highest in group I, the differences among the groups were not statistically significant $(p=0.074)$ (Table I).

In the BSA-1 group, the LPO was higher than in the BSA-2 group and it was $12.75 \pm 6.90 \mathrm{nmol} / \mathrm{ml}$ and $10.79 \pm 4.87 \mathrm{nmol} / \mathrm{ml}$, respectively $(p=0.023)$ (Figure 1).

We found a weak negative linear correlation between LPO and age $(r=-0.216, p<0.005$ (Figure 2)), body mass $(r=-0.153, p<0.05$ (Figure 3)), height $(r=-0.152, p<0.05$ (Figure 4)) and BSA $(r=-0.151, p<0.05$ (Figure 5)). We did not find a correlation between LPO and BMI (Figure 6).

A linear correlation between LPO and age was observed in girls $r=-0.251 \quad(p=0.0195)$, whereas it had no statistical significance in boys $(p=0.186)$. The strongest negative linear correlation between LPO and age was observed in
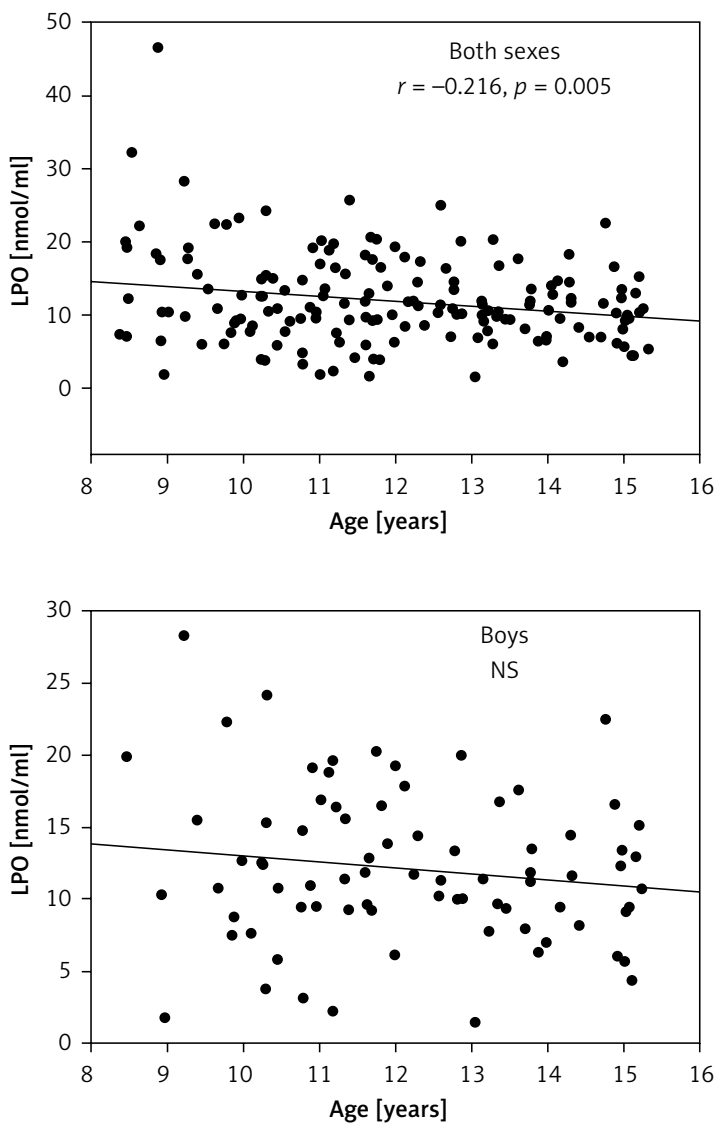

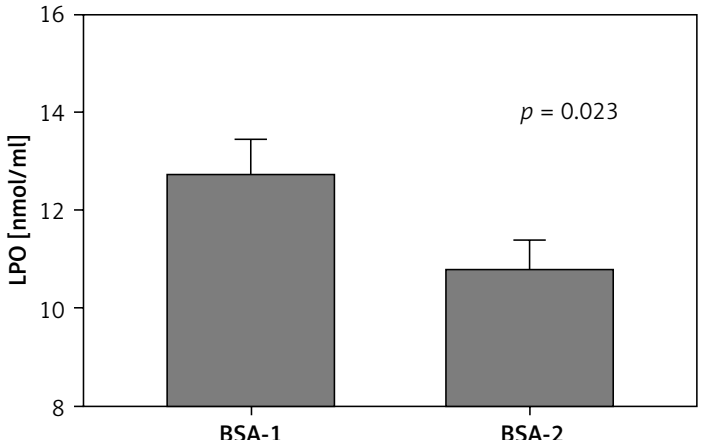

Figure 1. Comparison of lipid peroxidation (LPO) between groups body surface area 1 (BSA-1) and BSA-2

a group of girls up to 11 years of age $(r=-0.297$ with $p=0.0262$ ).

After stratification by gender, correlations between LPO and age, body mass, height and BSA were not statistically significant except for the aforementioned correlation between LPO and age in girls.

No correlation was found between the iodine supply and LPO. The UIC median was $100.6 \mu \mathrm{g} / \mathrm{l}$ (10.5-318.3), while mean \pm SD was $110.6 \pm 52.5$ $\mu \mathrm{g} / \mathrm{l}$.

However, in group III a positive correlation between LPO and thyroid volume was found, $r=0.268$ at $p=0.05$ (data not shown).

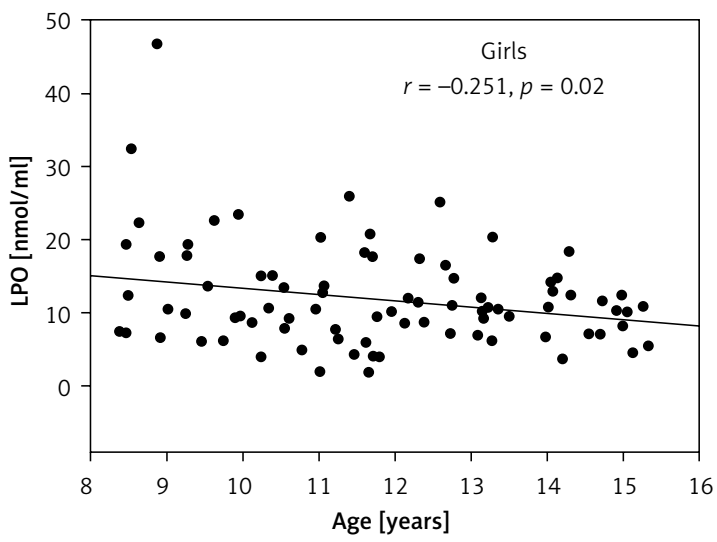

Figure 2. Correlation between lipid peroxidation (LPO) and age 

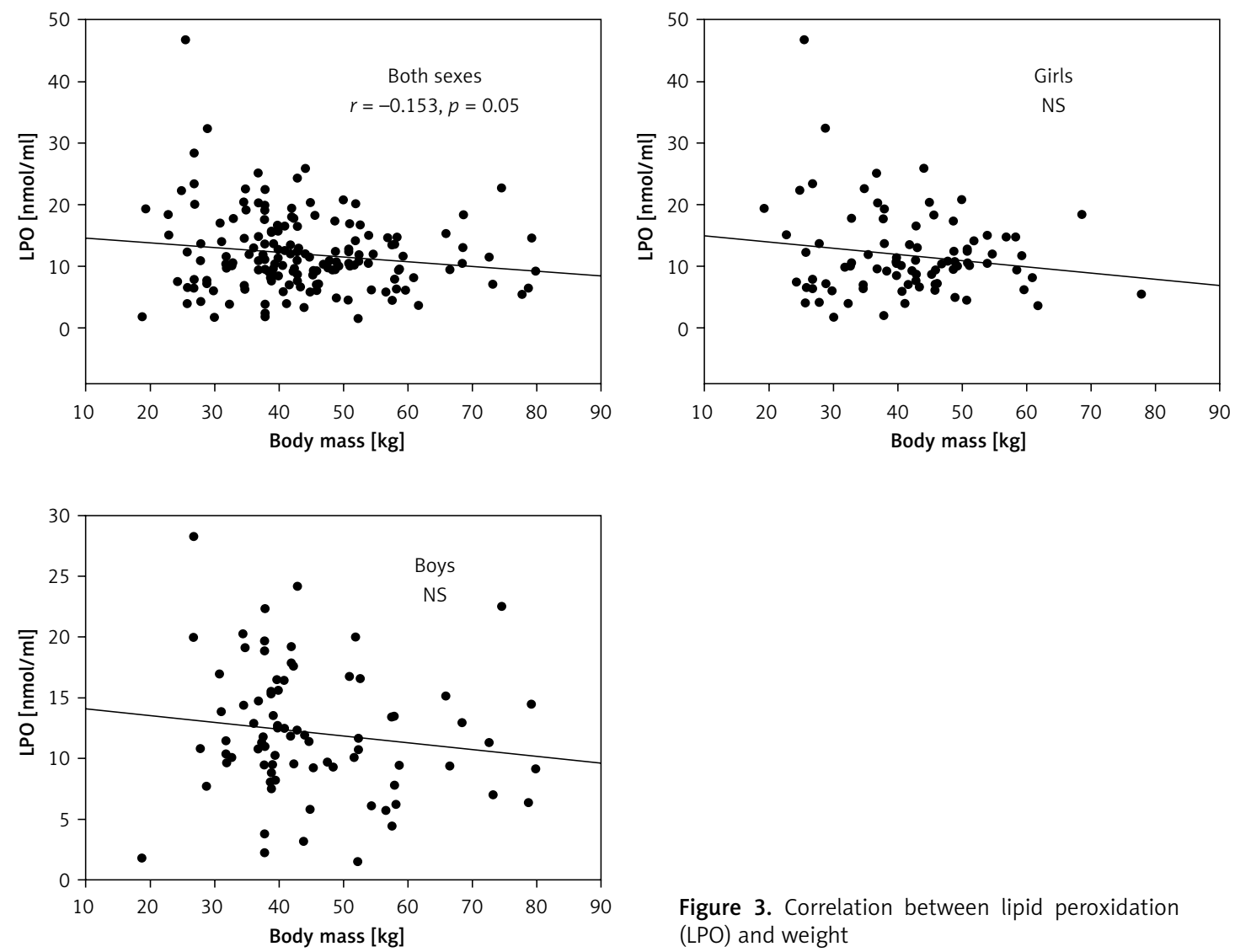

Figure 3. Correlation between lipid peroxidation (LPO) and weight
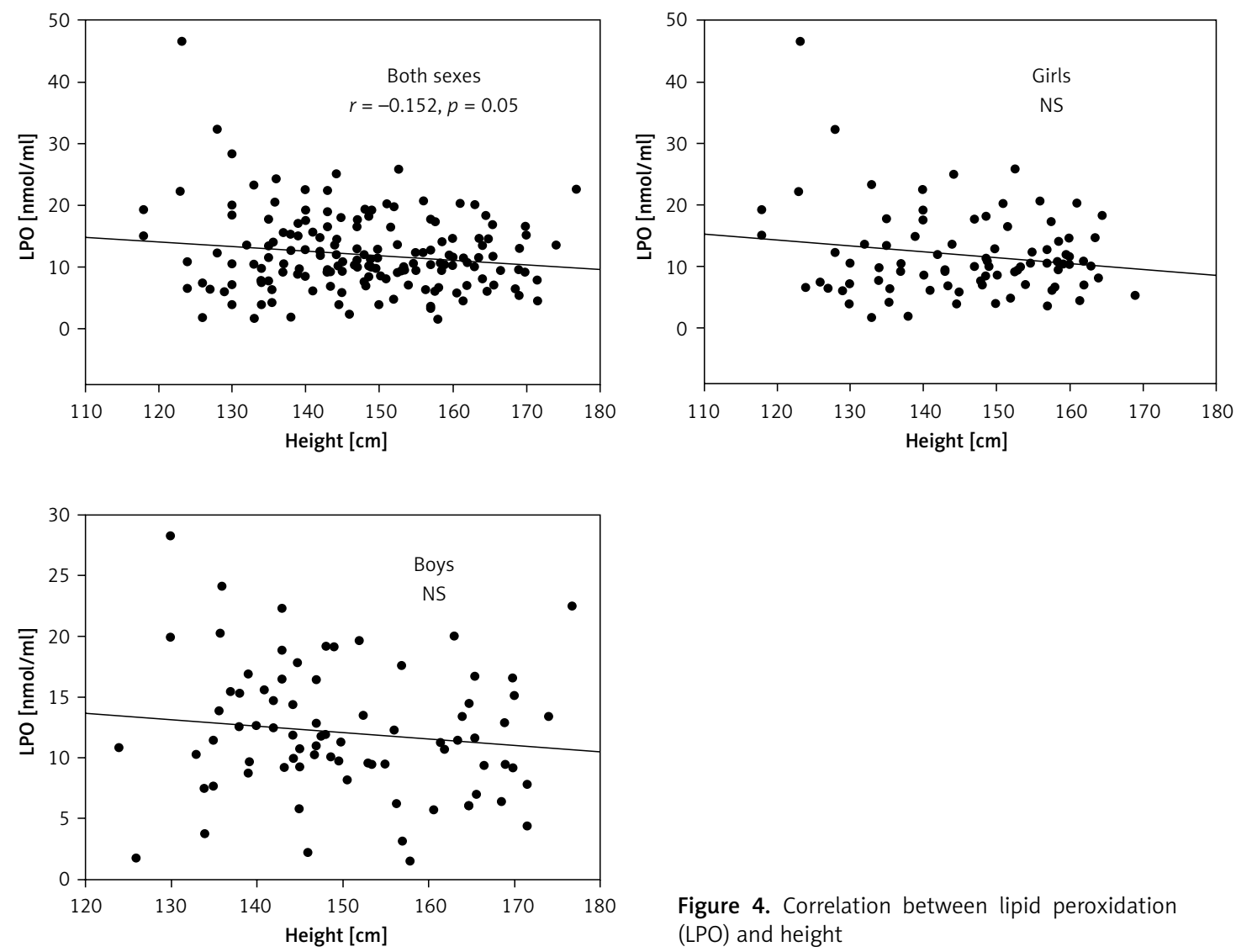

Figure 4. Correlation between lipid peroxidation (LPO) and height 

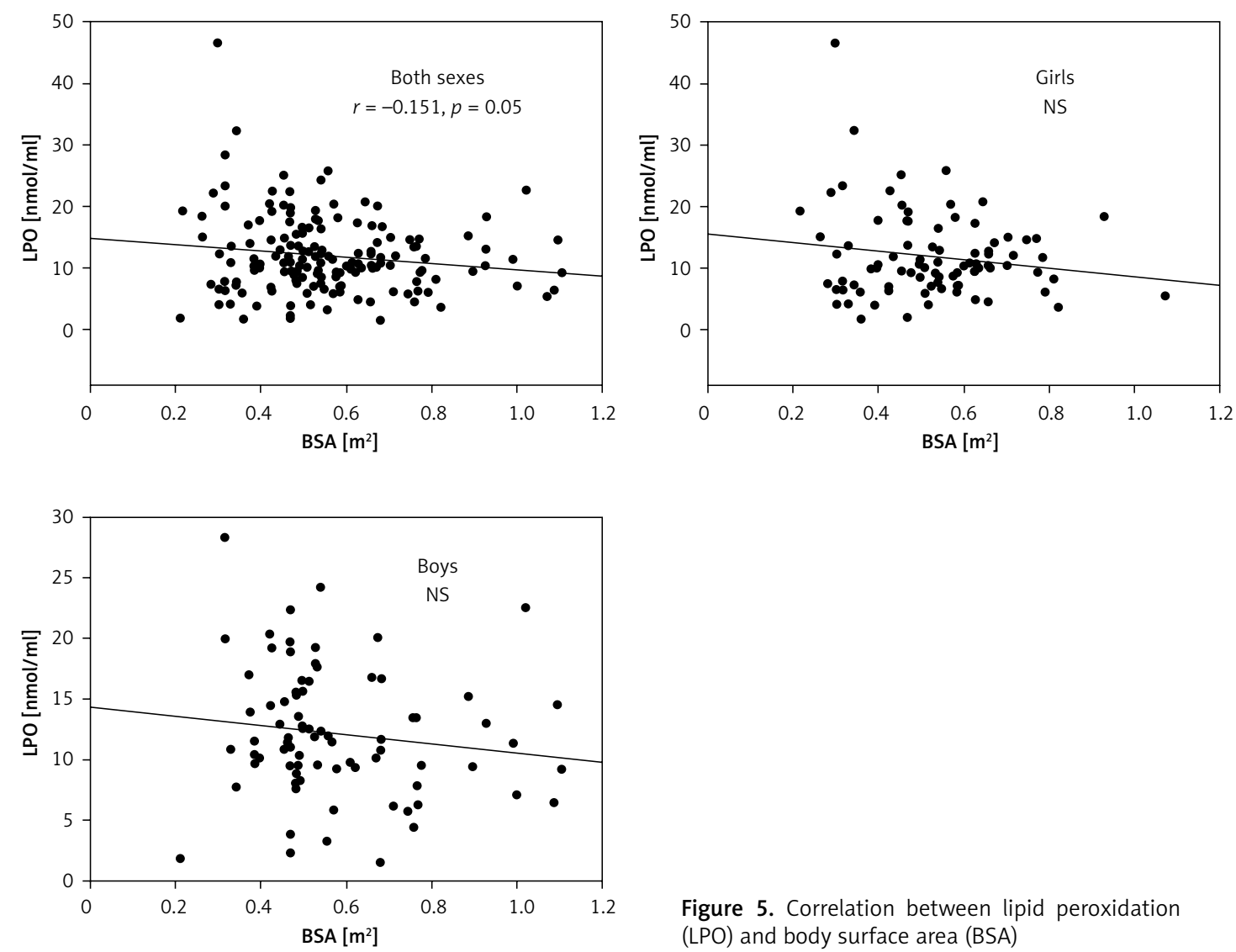

Figure 5. Correlation between lipid peroxidation (LPO) and body surface area (BSA)
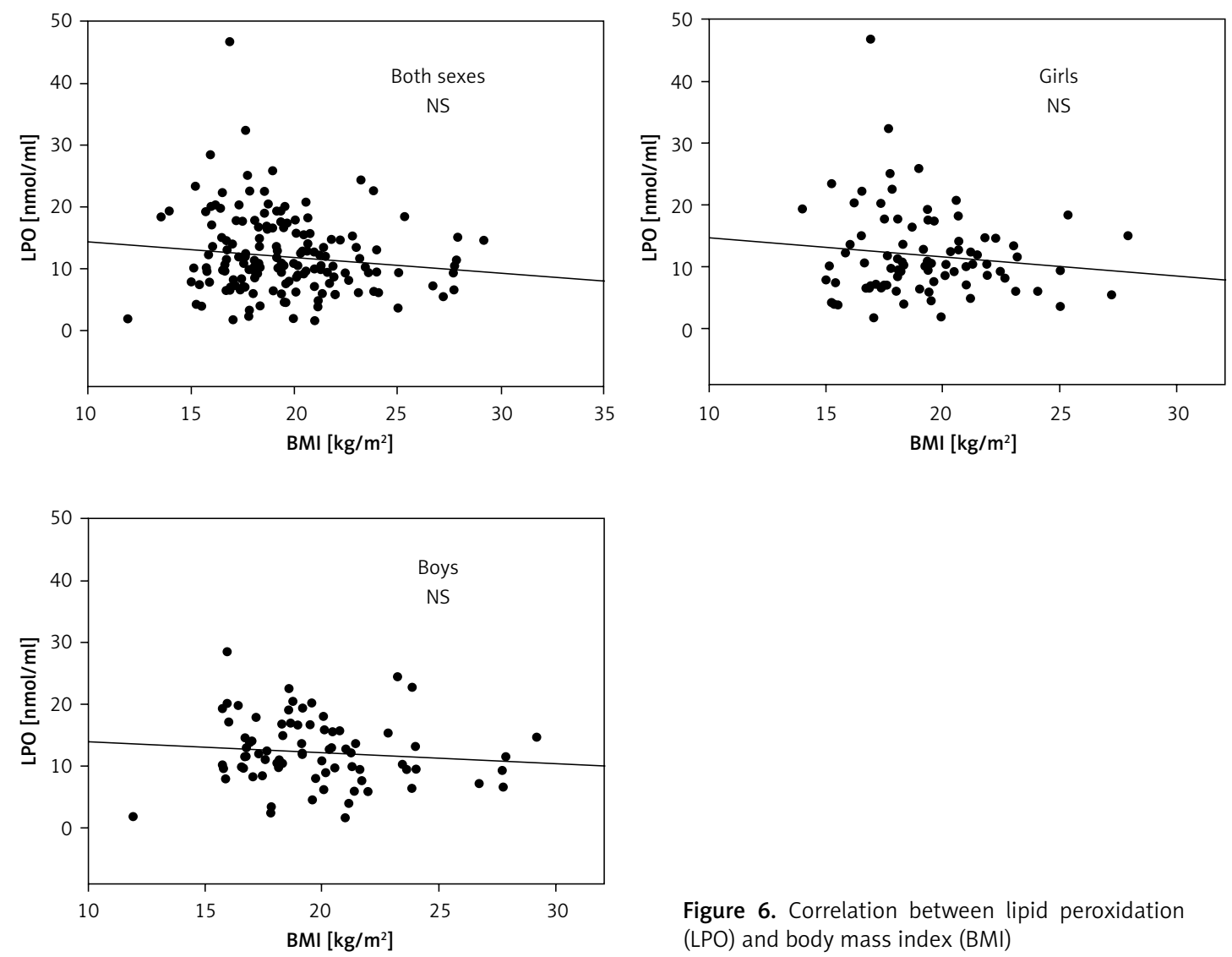

Figure 6. Correlation between lipid peroxidation (LPO) and body mass index (BMI) 


\section{Discussion}

The present study was an attempt to determine the relationship between iodine intake and the concentration of LPO. Expectedly, such a relationship was not confirmed statistically. Our research has shown that the supply of iodine in the children was correct; both mean and median UIC values were within normal limits. Because iodine is a strong factor affecting oxidative processes [18], it is of great importance to evaluate oxidative damage in the organism under conditions of proper iodine supply. Since the recommended iodine supply (measured as UIC) has been confirmed in the population of children enrolled in our previous study [15] and, at the same time, no clear relationship between urine LPO and UIC has been found in the present study, the above-mentioned precondition is met.

However, while the median value in the study group reflects iodine supply in the population in particular individuals, UIC is a parameter with too large variability to estimate the overall supply of iodine in a person based on just one measurement. Only $30 \%$ of iodine absorbed in the gastrointestinal tract is captured by the thyroid gland and the iodine biological half-life in human blood is $6 \mathrm{~h}$ [19]; therefore, it can be assumed that UIC depends mainly on the supply of iodine in the last few hours. Because of this, not capturing such a correlation does not exclude its existence. It would be very interesting to investigate UIC in the same person several times (preferably 10-fold) and to calculate the arithmetic mean from these studies [20]; this would be a more representative way to assess the supply of iodine in each individual.

While the value of UIC is an instantaneous value, the volume of the thyroid gland is a more constant parameter and indirectly may indicate iodine supply in the long-range period. A positive correlation between LPO and thyroid volume ( $r=0.268$ at $p=0.05)$ was observed among children from group III (12-13 years). Our observation is consistent with results obtained by Gérard et al. and Poncin et al. [9, 10]. In other age groups and in the analysis of the whole group of studied children, such dependencies were not detected.

It has been repeatedly documented that obesity is associated with increased damage to macromolecules, but almost all of studies in question were performed in adult populations. For example, a higher LPO level in blood serum [21] and in the epidermis [22] and a higher level of oxidative DNA damage [23] were found in adults with increased BMI. Unexpectedly, a negative relationship was found between urine LPO and BSA in the present study. However, in children, unlike in adults, in most cases increased BSA is not associated with obesity but just with physiological growth of the organism. Body mass index is a more relevant parameter reflecting overweight and obesity, and, indeed, it was the only value in our analysis in which such a negative correlation was not observed.

On the basis of data obtained from the study it can be said that the smaller the child is, the higher is the value of LPO. Though we were not able to demonstrate this by comparing the LPO in different age groups (no statistical significance), the comparison of children based on the BSA revealed that LPO in children with BSA $\leq 0.55$ is higher than in the case of children with BSA > 0.55 .

Furthermore, weak but consistent negative correlations were observed between LPO and age, weight, height and BSA. Our observations might indicate that the anti-oxidative barrier develops with age, like for instance the child's immunological system, reaching its peak at adolescence/early adulthood, and later it becomes weaker with age, which is commonly described in adults. However, Protano et al., who examined biomarkers of oxidative stress to nucleic acids in a group of healthy Italian children, described a different trend in their study [24]. They observed that the excretion of 8-oxoGuo in children increased with age, while it, unexpectedly, decreased with increasing BMI value. Undoubtedly, this issue requires further studies. A certain limitation of our study might be the relatively small number of subjects in younger age groups.

The novelty of our analysis is based on the fact that the LPO was determined not in the serum, but in the urine sample. Such assays are used infrequently, but with this type of biological material the method becomes non-invasive, which is especially important for studies performed in patients in developmental age. In order to better standardize the excreted substances it would be helpful to refer them to the creatinine excreted with urine.

In conclusion, anthropometric parameters of school-age children independently of age are negatively associated with oxidative damage to membrane lipids, whereas factors promoting goitrogenesis do not contribute to this process.

\section{Acknowledgments}

This study was financially supported by statutory funds from the Medical University of Lodz, Lodz, Poland (503/1-107-03/503-11-001).

\section{Conflict of interest}

The authors declare no conflict of interest.

\section{References}

1. Halliwell B. Free radicals and antioxidants: updating a personal view. Nutr Rev 2012; 70: 257-65.

2. Halliwell B. Cell culture, oxidative stress, and antioxidants: avoiding pitfalls. Biomed J 2014; 37: 99-105. 
3. Sedelnikova OA, Redon CE, Dickey JS, Nakamura AJ, Georgakilas AG, Bonner WM. Role of oxidatively induced DNA lesions in human pathogenesis. Mutat Res 2010; 704: 152-9.

4. Kryston TB, Georgiev AB, Pissis P, Georgakilas AG. Role of oxidative stress and DNA damage in human carcinogenesis. Mutat Res 2011; 711: 193-201.

5. Halliwell B, Gutteridge JMC. Free radicals in biology and medicine. $5^{\text {th }}$ ed. Oxford University Press, Oxford, United Kingdom 2015.

6. Karbownik M, Lewinski A. The role of oxidative stress in physiological and pathological processes in the thyroid gland; possible involvement in pineal-thyroid interactions. Neuro Endocrinol Lett 2003; 24: 293-303.

7. Kovacic P, Edwards C. Integrated approach to the mechanisms of thyroid toxins: electron transfer, reactive oxygen species, oxidative stress, cell signaling, receptors, and antioxidants. J Recept Signal Transduction Res 2010; 30: 133-42.

8. Poncin S, Gérard AC, Boucquey M, et al. Oxidative stress in the thyroid gland: from harmlessness to hazard depending on the iodine content. Endocrinology 2008; 149: 424-33.

9. Gérard AC, Poncin S, Audinot JN, Denef JF, Colin IM. lodide deficiency-induced angiogenic stimulus in the thyroid occurs via HIF- and ROS-dependent VEGF-A secretion from thyrocytes. Am J Physiol Endocrinol Metab 2009; 296: E1414-22.

10. Poncin S, Van Eeckoudt S, Humblet K, Colin IM, Gérard AC. Oxidative stress: a required condition for thyroid cell proliferation. Am J Pathol 2010; 176: 1355-63.

11. Synowiec E, Wójcik KA, Czubatka A, et al. Lack of association between polymorphisms of the DNA base excision repair genes MUTYH and hOGG1 and keratoconus in a Polish subpopulation. Arch Med Sci 2015; 11 : 1101-10.

12. Qin WS, Deng YH, Cui FC. Sulforaphane protects against acrolein-induced oxidative stress and inflammatory responses: modulation of Nrf-2 and COX-2 expression. Arch Med Sci 2016; 12: 871-80.

13. Szychta P, Zadrozny M, Lewinski A, Karbownik-Lewinska $M$. Increased oxidative damage to membrane lipids following surgery for breast cancer. Neuro Endocrinol Lett 2014; 35: 602-7.

14. Lewandowski KC, Stojanovic N, Press M, Tuck S, Lewiński A, Karbownik-Lewińska M. Raised concentrations of lipid peroxidation products (LPO) in pregnant women with impaired glucose tolerance. Ann Agric Environ Med 2014; 21: 429-34.

15. Zygmunt A, Adamczewski Z, Wojciechowska-Durczyńska K, et al. Evaluation of efficacy of iodine prophylaxis in Poland based on the examination of schoolchildren living in Opoczno Town (Lodz Voivodship). Thyroid Res 2012; 5: 23.

16. World Health Organization: Physical status: the use and interpretation of anthropometry. Report of a WHO Expert Committee. World Health Organ Tech Rep Ser 1995; 854: 1-452.

17. Sandell EB, Kolthoff IM. Microdetermination of iodine by catalytic method. Microchim Acta 1937; 1: 9-25.

18. Karbownik-Lewińska M, Kokoszko-Bilska A. Oxidative damage to macromolecules in the thyroid - experimental evidence. Thyroid Res 2012; 5: 25.

19. Kramer GH. Retention of iodine in the body: biological half-life of iodine in the human body. In: Comprehensive Handbook of lodine. Nutritional Biochemical, Pathological and therapeutic aspects. Preedy VR, Burrow GN,
Watson RR (eds). Academic Press is an imprint of Elsevier 2009

20. König F, Andersson M, Hotz K, Aeberli I, Zimmermann MB. Ten repeat collections for urinary iodine from spot samples or 24-hour samples are needed to reliably estimate individual iodine status in women. J Nutr 2011; 141: 2049-54.

21. Kokoszko A, Karbownik M, Lewiński A. Increased lipid peroxidation in growth hormone-deficient adult patients. Neuro Endocrinol Lett 2006; 27: 225-30.

22. Szokalska K, Stepniak J, Karbownik-Lewinska M. Lipid peroxidation evaluated in epidermis exfoliated during microdermabrasion is a reliable marker of oxidative stress related to obesity. J Eur Acad Dermatol Venereol 2016; 30: 1429-31.

23. Karbownik-Lewinska M, Szosland J, Kokoszko-Bilska A, et al. Direct contribution of obesity to oxidative damage to macromolecules. Neuro Endocrinol Lett 2012; 33 : 453-61.

24. Protano C, Andreoli R, Mutti A, Petti S, Vitali M. Biomarkers of oxidative stress to nucleic acids: background levels and effects of body mass index and life-style factors in an urban paediatric population. Sci Total Environ 2014; 500-501: 44-51. 